\title{
CLINICAL LECTURES
}

ON

\section{THE DIAGNOSIS OF TUMOURS OF THE BREAST.}

By THOMAS BRYANT, EsQ., F.R.C.S.,*

Assistant-Surgeon, Guy's Hospital.

LECTURE I.

General Remarks. Points to be noted in Cases of Tumour.

Gentlemen,-I purpose during the present clinical season directing your attention to the question of the diagnosis of disease; for, in the practice of a profession like our own, there are few things which require more careful observation, accurate thought, and scientific study, than the phenomena of symptoms.

The human frame exhibits marvellous complexity. The expressions of its physiological actions and pathological processes are most varied; and it is owing to these truths that we cannot with absolute accuracy describe any symptom as being positively indicative of a certain condition of organ, as being pathognomonic of a definite disease.

The science of medicine labours under the disadvantages of all inexact sciences: it rests on probabilities; nevertheless, it possesses the advantage of suggesting in its daily practice strange and varied problems, requiring for solution sound and matured thought, analysis, and judgment. In the diagnosis of disease, however, a fair degree of certainty is to be attained, a certainty based on probabilities varying in degree in every individual case. In some cases the probabilities are so feeble that our diagnosis must be regarded as uncertain; in others the probabilities are so numerous and their convergency so great that certainty is established. Between these extremes lie many degrees, and it is in the power of correctly estimating these degrees that the different minds of men are found to vary: the balancing or judging power of the mind fairly determining the diagnostic capabilities of its possessor; a correct diagnosis resting upon the accurate appreciation of the value of the facts or symptoms presented by the individual case.

Diagnosis is therefore most important, and requires careful study. It is, in fact, the keystone of the arch upon which successful practice is to be raised. Nothing can be done till this is laid. We can adopt no scientific treatment whilst we are ignorant of the exact nature of the case.

I purpose, therefore, to devote my attention to the question of diagnosis of disease. I shall take up those subjects which I know from experience to be most difficult. The diagnosis of tumours will first claim our consideration; especially tumours of the breast and testicle. The diagnosis of diseases of the joints will probably terminate our course.

I must ask you to give me close attention, for, I assure you, the subjects present many difficulties, and you will obtain profit from my observations in proportion to the closeness with which you follow them. We shall regard these subjects purely from a clinical point of view, and I shall present to you the line of argument that a surgeon employs when a patient informs him that he or she is suffering from an affection of the breast, of the testicle, or of a joint.

To-day's lecture will be occupied by the consideration of the subject of "Tumour of the Breast." Now, what are the means at the surgeon's disposal, by which the diagnosis of disease is to be made? What mental and physical modes of investigation would he adopt in his attempt to form an opinion of the nature of the affection? $\mathrm{He}$ would first of all avail himself of the information elicited from the history of the case ; facts furnished by inspection of the part would next claim his attention, and lastly the facts revealed by manual examination. When these three modes of investigation lead to the same conclusion, the diagnosis may be pronounced to be tolerably certain ; when each tends to suggest the presence of different conditions, the diagnosis will be rendered difficult; the degree of difficulty varying widely in different cases. But what I wish you to remember, and not only to remember but to make a rule of your professional life, is, that you should invariably employ all these three modes of investigation in every case that comes under your notice. If you dispense with one, or trust to one more than another, you will constantly be falling into error, constantly throwing away an opportunity of forming a correct opinion as to the nature of a case, when a little care, a little method, would have kept you right. The surgeon, on going over the history of a case, ascertaining when the symptoms of disease first appeared, when others manifested themselves, and how they were developed, almost instinctively forms an opinion as to the nature of the affection he has before him; when he has looked with a critical eye at the part affected, marked the symptoms which are manifest to his sight, and finds the opinion he had formed on learning the history and progress of the disease confirmed by the external or visible appearances of the part, the correctness of his original diagnosis without doubt becomes more probable; and lastly, when the opinion thus formed is confirmed by the indications supplied by a careful manual examination, the scientific accuracy of the diagnosis can remain no longer a subject of doubt. It is by this method of investigation alone that accuracy can be obtained, and scientific diagnosis arrived at.

I now purpose considering the special points to be observed in recording the history of a tumour of the breast, and estimating their value ; but, before doing so, it will be well to remind you of the different affections to which the breast is liable. I shall make a simple classification which will answer our purpose: 1 . Inflammatory tumours; 2. Innocent or adenoid tumours; 3. Malignant tumours.

I will, however, dismiss from our consideration the acute inflammatory affection of the breast, for the diagnosis of such a case should be no subject of difficulty; in that affection all the local and general symptoms of inflammation will be present, as swelling, heat, pain, and redness, and probablv the inflammation will terminate in suppuration. No difficulty in the diagnosis is likely to present itself. The subject of chronic inflammatory tumours, whether terminating in suppuration or not, will, however, come under our consideration, for it is here that great difficulty in the diagnosis is often experienced, and the greatest errors in practice often perpetrated.

Let us consider for a few minutes the pathological points of difference between innocent and cancerous tumours, and in what ways clinically they differ, for clinical observation and pathological knowledge should invariably go hand in hand. Amongst other points of difference, the following is without doubt the most important:-"Simple tumours separate tissues in their growth, but never infiltrate; cancerous as a rule infiltrate, and rarely separate."

No more important point can be brought forward to aid the surgeon in his diagnosis of a tumour than the above. For a simple or innocent tumour, however long it may be in growing, and however large a size it may attain, will never do more than separate the parts between and beneath which it may be developed. The bones may even be absorbed by its presence, but they will never be involved; and the skin may be so stretched and attenuated by its distension as to ulcerate and burst, but it will never be infiltrated with the tumour's elements. This fact is well exemplified by a close examination of the margin of a cutaneous opening, the result of over distension from a simple tumour, for it will appear as if cleanly cut, or rather punched, at its edges, but never thickened or diseased.

With the cancerous tumour, however, a very different condition has to be described, for a cancer has the peculiar property of freely infiltrating all the tissues upon which it presses. As the tumour approaches the surface, the skin will become involved; at first, it may appear as if drawn down to it, then puckered, and afterwards as though glued to its surface; at a later stage of the disease, the skin will become infiltrated with the cancerous elements, and to the finger feels firm, fibrous or tuberculated, and when ulceration has commenced, the edges of the skin will be palpably indurated, thickened, and infiltrated with cancerous products. The contrast between these different conditions of integument in the two classes of tumours is most marked, and very important, and forms a most valuable means of diagnosis in the extreme stage of simple and malignant tumours, whether of the breast or of any other part.

There is one other point of difference to which I would wish to draw your attention, and it is this: "That simple or innocent tumours affect the patient solely through their local influence, and have no tendency to multiplication in other tissues, nor to involve the absorbents with which they are connected."

"Cancerous tumours not only affect the patient through their local influence, but have a marvellous tendency to multiplication in any part of the body, more particularly in the internal, and never exist for any period without implicating the lymphatics of the part with which they are connected"

This difference between the two classes of cases is most important, and forms a valuable means of diagnosis even in an early condition of disease; for in a case of tumour, the nature of which is doubtful from both its local and general conditions, the presence or absence of an indurated absorbent gland, not an inflamed one, will weigh down the balance of doubt, and tend more strongly than anything else to solve the problem; for it is as rare to meet with indurated glands in a simple tumour, as it is rare to miss them in a cancerous. 